University of New Hampshire

University of New Hampshire Scholars' Repository

6-3-2013

\title{
Characterization of optical communication in a leader-follower unmanned underwater vehicle formation
}

\author{
Firat Eren \\ University of New Hampshire, Durham, Firat.Eren@unh.edu \\ Shachak Pe'eri \\ University of New Hampshire, Durham, shachak.peeri@unh.edu \\ May-Win Thein \\ University of New Hampshire, Durham
}

Follow this and additional works at: https://scholars.unh.edu/ccom

Part of the Computer Sciences Commons, and the Oceanography and Atmospheric Sciences and Meteorology Commons

\section{Recommended Citation}

F. Eren, S. Pe'eri, and M.-W. Thein, "Characterization of optical communication in a leader-follower unmanned underwater vehicle formation," in Ocean Sensing and Monitoring V, SPIE-Intl Soc Optical Eng, 2013.

This Conference Proceeding is brought to you for free and open access by the Center for Coastal and Ocean Mapping at University of New Hampshire Scholars' Repository. It has been accepted for inclusion in Center for Coastal and Ocean Mapping by an authorized administrator of University of New Hampshire Scholars' Repository. For more information, please contact Scholarly.Communication@unh.edu. 


\title{
Characterization of optical communication in a leader-follower unmanned underwater vehicle formation
}

\author{
Firat Eren ${ }^{1}$, Shachak Pe'eri ${ }^{2}$ and May-Win Thein ${ }^{1}$ \\ ${ }^{1}$ Department of Mechanical Engineering, University of New Hampshire, Durham, NH 03824 \\ ${ }^{2}$ Center for Coastal and Ocean Mapping, University of New Hampshire, Durham, NH 03824
}

\begin{abstract}
As part of theresearchto development an optical communication design of a leader-follower formation between unmanned underwater vehicles (UUVs), this paper presents light field characterization and design configuration of the hardware required to allow the use of distance detection between UUVs. The studyspecifically is targeting communication between remotely operated vehicles (ROVs). As an initial step in this study, the light field produced from a light source mounted on the leader UUV was empirically characterized and modeled. Based on the light field measurements, a photo-detector array for the follower UUV was designed. Evaluation of the communication algorithms to monitor the UUV's motion was conducted through underwater experiments in the Ocean Engineering Laboratory at the University of New Hampshire. The optimal spectral range was determined based on the calculation of the diffuse attenuation coefficients by using two different light sources and a spectrometer. The range between the leader and the follower vehicles for a specific water type was determined. In addition, the array design and the communication algorithms were modified according to the results from the light field.
\end{abstract}

Key words:Unmanned underwater vehicle, optical communication, light attenuation, water clarity, simulation

\section{INTRODUCTION}

Unmanned underwater vehicles (UUVs) play a major role in deep oceanic applications, such as underwater pipeline and cable inspection, bathymetry exploration as well as in military applications such as mine detection, harbor monitoring and anti-submarine warfare[1-5]. These applications mostly take place in deep sea environment and include heavy duty tasks that may take long time periods and therefore, are not suitable to be performed by divers. The use of multiple UUVs simultaneously for these types of tasks that can be controlled by one operator has been a research interest as a cost-efficient solution for large-scale surveys with minimum time. The focus of this paper is optical communication between multiple UUVs in a specified formation, such as a leader-follower formation. In typical UUV leader-follower formation systems such as [6-8] utilize acousticsas the main method of communication between the vehicles. However, hardware (e.g., acoustic transducers) can be very costly and are also limited by the logistics required in modifying the leader UUV [9]. Optical communication modules can provide an alternative costefficient approach. In this research, we propose an optical communication link between the UUVs to form a formation. UUVs already use light sources to illuminate the seafloor and in this paper this hardware is used as a beacon for aligning follower UUVs.

Preliminary work for this study included the development of a control design for distance detection of UUV usingoptical sensor feedback in a Leader-Follower formation [10]. The distance detection algorithms were designed to detect translational motion above water utilizing a beam of light for guidance. The light field of the beam was modeled usinga Gaussian function as a first-order approximation. This light field model was integrated into nonlinear UUV equations of motion for simulation to regulate the distance between the leader and the follower vehicles to a specified reference value. A prototype design of a photo-detector array consisting of photodiodes was constructed and tested above water. However, before an array can be mounted on the bow of the follower UUV, a better understanding of the underwater light is needed. The proposed system is based on detecting the relative light intensity changes on the photodiodes in the array. The size of the array strictly depends on the size of the ROV. This paper provides an overview on the experiments and simulations conducted to adjust the control design for underwater conditions. Underwater light is attenuated due to the optical characteristics of the water, which are 
constantly changing and are not uniformly distributed. As a result, applying distance detection algorithms underwateradds complexity and reduces operational ranges. Accordingly, the operation distance between the UUVs was limited to a range between 4.5 to $8.5 \mathrm{~m}$ for best performance. Experimental work in this study was performed in the Tow and Wave Tank at the Ocean Engineering facilities, University of New Hampshire (UNH).

\section{THEORETICAL BACKGROUND}

The basic concept for optical communication in this study is based on the relative intensity measured between the detectors within the photo-detector array mounted on the follower ROV. In addition to the beam pattern produced by the light source, the intensity of light underwater follows two basic concepts in ocean optics [11]:The inverse square law and Beer-Lambert law.

\subsection{Beam pattern}

The light field emitted from a light source can be modeled with different mathematical functions. In addition, there are a variety of light sources that can be used underwater that differ in their spectral irradiance (e.g., halogen, tungsten, and metal-halide). The spectral characteristics of the light source are an important issue that affects the illumination range, detector type and the detection algorithms. As in the case of the light sources, the photodetectors also have a spectral width in which their sensitivity is at a maximum value. By determining the spectral characteristics of the light source, it is possible to select the detector and filters for the photodetector array. We assume that the beam pattern can be modeled using a Gaussian function. This representation is valid for a single point light source. The Gaussian model used in this study can be representedasfollows[12]:

$$
I(\theta)=A * \exp \left(-B * \theta^{2}\right)
$$

In Equation $1, I$ is the intensity at a polar angle, $\theta$, where the origin of the coordinate system is centered around the beam direction of the light source. $A$ and $B$ are constants that describe the Gaussian amplitude and width respectively.

\section{$2.2 \quad$ Inverse square law}

According to the inverse square law, the intensity of the light is inversely proportional to the inverse square of the distance:

$$
I=\frac{S}{4 \pi r^{2}}
$$

where $I$ is the intensity at $\mathrm{r}$ distance away from the source and $\mathrm{S}$ is the light field intensity at the surface of the sphere. Thus, the ratio of the light intensities at two different locations at the same axis can be expressed in Equation 3.

$$
\frac{I_{1}}{I_{2}}=\frac{\frac{S}{4 \pi r_{1}^{2}}}{\frac{S}{4 \pi r_{2}^{2}}}=\left(\frac{r_{2}}{r_{1}}\right)^{2}
$$

The light field $S$ generated by a light source is assumed to show uniform illumination characteristics in all directions.In addition, the light intensity is such that the light source is assumed to be a point source and that its intensity is not absorbed by the medium. It should also be noted that although the inverse square law is the dominant concept in the development of control algorithms, for this research this is not the only dominant optical mechanism that affects the light passingin water. As the light travelsthrough water, its rays also get absorbed by the medium according to Beer-Lambert law.

\subsection{Beer-Lambert law}

Beer-Lambertlaw states that radiance at an optical path length $l$ in a medium decreases exponentially depending on the optical length, $l$, the angle of incidence, $\theta$, and the attenuation coefficient, $K$ [13]:Beer-Lambertlaw describes the 
light absorption in a medium under the assumption that an absorbing, source free medium is homogeneous and scattering is not significant. When the light travels through a medium, its energy is absorbed exponentially[13].

$$
L(\zeta, \hat{\xi})=L(0, \hat{\xi}) \exp \left(-\frac{\zeta}{\mu}\right)
$$

where $L$ denotesthe radiance, $\zeta$ the optical depth, $\hat{\xi}$ the direction vector, and $\mu$ denotes the light distribution as a function of angle $\theta$ such that:

$$
\mu=\cos \theta
$$

Defining a quantity $l$, (i.e. the optical path length in direction $\mu$ ),

$$
d l \equiv \frac{d \zeta}{\mu}=\frac{K(z) d z}{\mu}
$$

where $K(z)$ is the total beam attenuation coefficient and $d z$ is the geometric depth.

In this study, the experimental setup is built such that the incidence angle $\theta$ is zero. As a result, combination of Equations 3 and 5 results in the Equation 6.

$$
L(\zeta, \hat{\xi})=L(0, \hat{\xi}) \exp (-K(z) d z)
$$

\section{EXPERIMENTAL SETUP}

Experimental work in this study was performed in order to evaluate a proposed hardware design which was based on ocean opticsand the hardware restrictions for a given ROV system. The experiments included beam diagnostics, spectral analysis and intensity measurements from several light sources.These experiments were conducted in the Tow and Wave Tank at theOcean Engineering facilities, University of New Hampshire(UNH). The UNH tow tank has a tow carriage that moves on rails. Alight source wasmounted on a rigid frame to the wall in the tow tank and alight detector wasplaced underwater connected to a tow carriage(Figure 1). This experimental setup is based on the design configured by [11]. To characterize the interaction between the light source and the light array a 50 Watt halogen lamp powered by $12 \mathrm{~V}$ power source is used. For the detector unit, a spectrometer (Ocean Optics Jaz)was used to characterize the underwater light field. These empirical measurements were used to adjust the detection algorithms and will be also used in the design of the photo-detector array.The light source in the tank simulates a light source that is mounted on the crest of the leader ROV. The design of the photo-detector array simulates the array that will be mounted on the bow of the follower ROV. The photo-detector array design depends on the size of the ROVand the light field produced by the light source mounted on the leader ROV. In this case, the size for an optical detector module was kept at $0.4 \mathrm{~m}$, which is the width dimension of the UNHROV as a test platform, a small observation class ROV which will be used as a future test platform. 


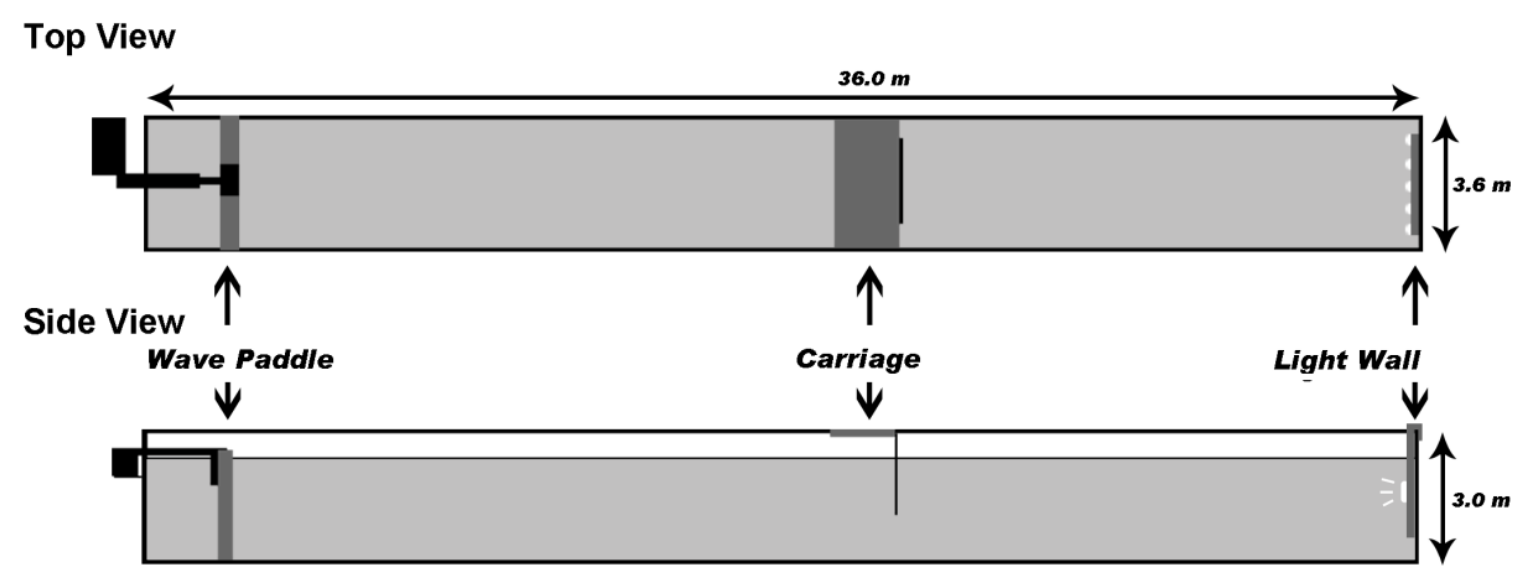

Figure 1: Experimental schematic of UNH tow tank [11]

Translational experiments in 1-D and 3-D (i.e., motion along and perpendicular to the center beam of the light source) were conducted in air and in water. The goals for the 1-D experiments were to characterize the spectral properties of the water and to determine the best spectral ranges for optical communication between the ROVs. In the underwater experiment, a submerged fiber optic cable with a collimator was connected to the spectrometer and was vertically aligned based on the peak value of radiance emitted from the light source. This alignment is considered the illumination axis (z-axis). The radiance emitted from the light source through the water column was empirically measured by the spectrometer at distances ranging from $4 \mathrm{~m}$ to $8 \mathrm{~m}$ at $1 \mathrm{~m}$ increments. It is important to note that the distances were measured from the tow carriage to the wall of the tank and an additional $0.5 \mathrm{~m}$ offset distance was added in the calculation to take into account the offset mounting of the light and spectrometer with respect to the wall of the tank and the tow carriage. The spectrometer was configured to average 40 samples with anintegration time of 15 milliseconds. A $2^{\circ}$ collimator was used to restrict the field of view collected by the spectrometer and to avoid the collection of stray light rays reflecting off the tank walls or from the water surface.

The experimental setup in air was very similar, where the spectrometer was mounted on a tripod and aligned to the peak value of radiance,the illumination axis (z-axis). Because suchlight sources produce heat at high temperatures (up to $700 \mathrm{C}$ ) that can damage the waterproof fixing, the experimental setup in air required thatthelight source be submerged in an aquarium during operation. Similar to theunderwater experiments, the same distances between the light source and the spectrometer, including the offsets, were maintained.

The 3-D translational underwater experiments utilized the same setup as that of the underwater 1-D experimentswhere additional radiance measurements were conducted along a normal axis ( $\mathrm{x}$-axis) that is located on a plane normal to the illumination axis (z-axis).The 3-D translational experiment maintainedthe same distances along the illumination axis between the light source and the spectrometer (i.e., $4 \mathrm{~m}$ to $8 \mathrm{~m}$ ), where additional measurements were conducted along the normal axis at $0.1 \mathrm{~m}$ increments ranging from $0 \mathrm{~m}$ to $1 \mathrm{~m}$. As mentioned in Section 2, it isassumed that the light source is producing a beam pattern that can be modeled using a Gaussian function. Accordingly, we assume that the radiance measurements along the normal axis are symmetric in all directions. The experimental setups for 3-D underwater experiments can be seen in Figure 2. 


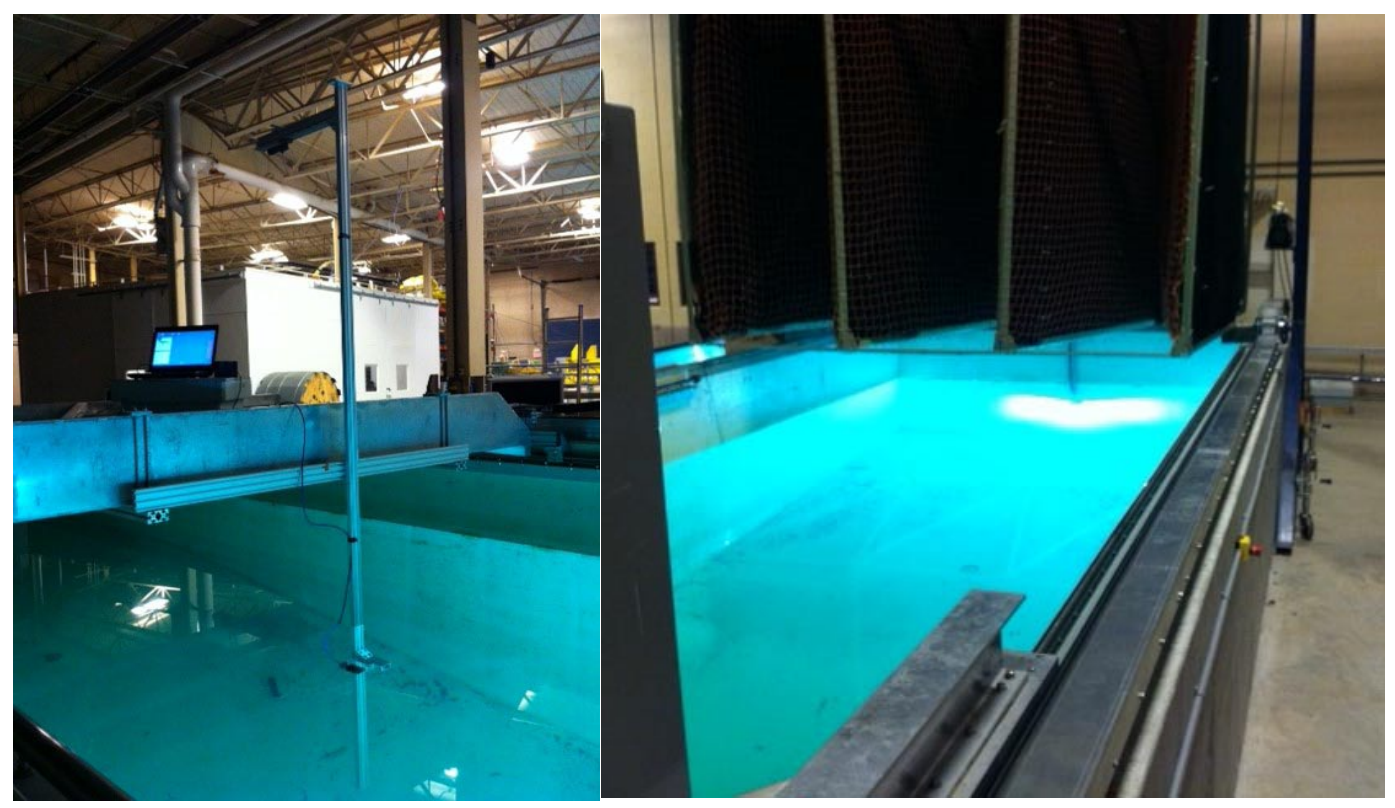

Figure 2: Experimental Setup for translational 3-D underwater experiments. (Left image) detector unit that includes a submerged fiber optic cable with a collimator that was connected to the spectrometer. (Right image) transmitting unit mounted to the wall of the tank.

\section{RESULTS}

Light attenuation underwater causes a significant loss of radiant energy over increasing distances. The diffuse attenuation coefficient, $K$, is used as a parameter to calculate the decreased amount of energy from the light source to the target. In this study, the diffuse attenuation coefficient is used to determine the spectral range of the light source and determine the photo-detector types that can be utilized in the array. For successful optical communication up to ranges of $9 \mathrm{~m}$, the ideal spectral ranges should be maintained such that the diffuse attenuation coefficient values are smaller than $0.1 \mathrm{~m}^{-1}$. At this distance, the signal loses about half its energy. As a first-order approximation, the diffuse attenuation coefficient values are assumed constant throughout the water column. This assumption reduces the number of parameters used in the distance detection algorithms and the processing time used in future controls applications. In the study, the diffuse attenuation coefficient values are calculated for a 50W light source. In Figure 3, the percentage loss curve for various distances is shown.

Diffuse attenuation was calculated based on Equation 6. Measurements taken at a specific distance in water and in air are compared in order to account for the inverse square law. The light that travels in air also undergoes diffuse attenuation but it is ignored in this case. These values suggest that the UNH wave tank, where the experiments were conducted, contains algae and dissolved matter. The study results suggest that 500-550 nm band-pass filters in the range shouldbe used in the detector unit to provide better performance of the distance detection algorithms. 


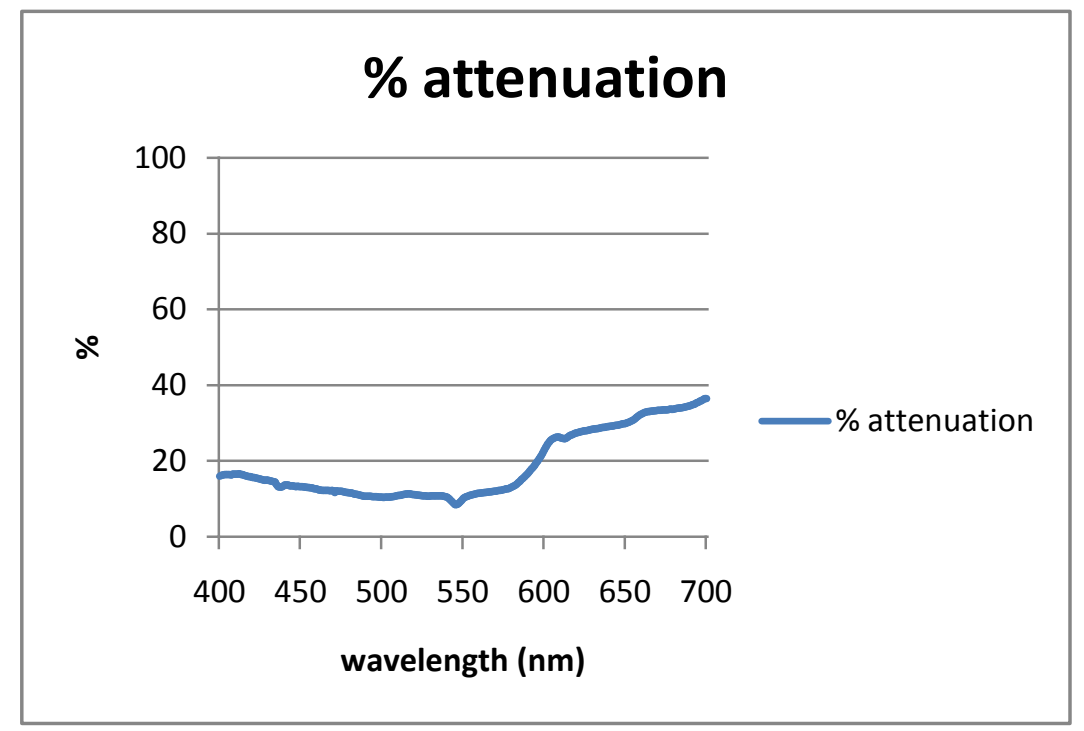

Figure 3: Percent attenuation graph. This graph shows the light percent attenuation per meter. It is seen that the spectral range between 500-550nm undergoes the least attenuation at any given distance.

Based on the light attenuation results, the distance between the leader and the follower vehicles was calculated. The experimental results (Figure 4) show that the performance of the algorithms in the UNH water tank is expected to decrease after $8.5 \mathrm{~m}$. Beyond this range, the light intensity falls into the background noise level (i.e., $<20 \%$ ).

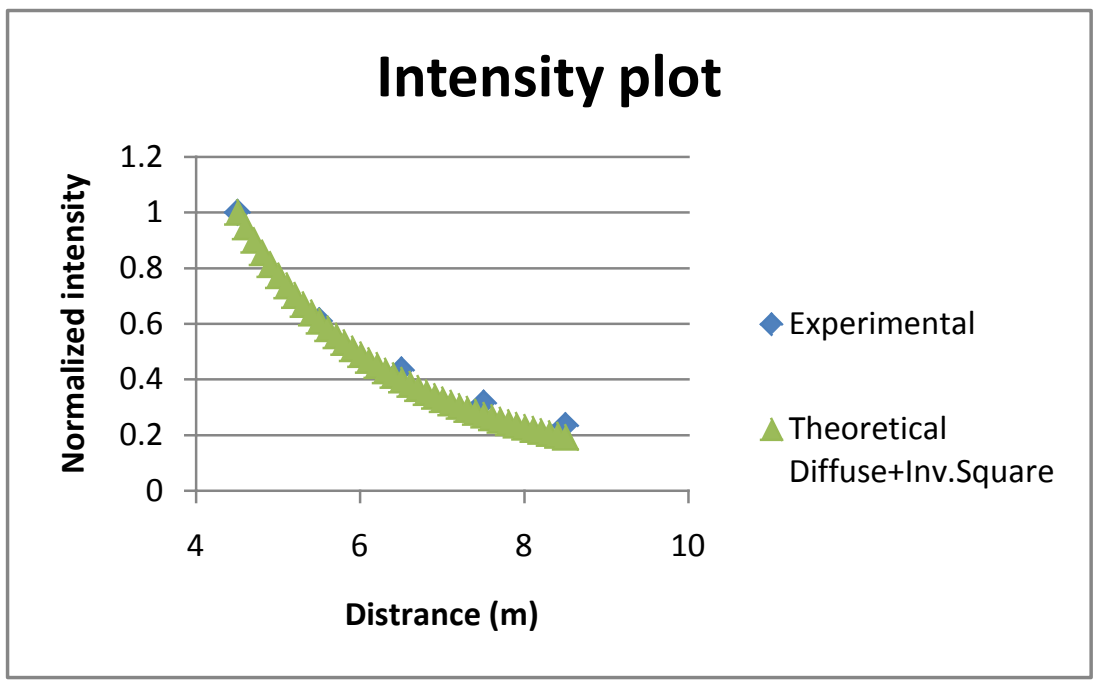

Figure 4: The intensity vs. distance plot. The intensity readings are collected between 500-550nm and averaged. In this plot, the experimental values are compared with the theoretical. Blue diamonds represent the experimental data and the green triangles represent the theoretical calculations from taking the inverse square and Beer-Lambert laws. The readings are normalized. The measurement at $4.5 \mathrm{~m}$ was used as the reference measurement to normalize the intensity. 
The light profile calculated from the 3-D experiments agrees with the assumption that the pattern of the light beam can be described using a 2-D Gaussian fit (Figure 5). Using a 50\% intensity decrease as a threshold, the effective beam radius from the center (i.e., the illumination axis) is $0.3 \mathrm{~m}$. Another key finding obtained from the 3-D experiments, is the dimensions of the light detector array. It can be seen that if the length of the array is kept at $0.6 \mathrm{~m}$, then different light detector elements can detect the light intensity change, which is useful information for control algorithms. It should be stated that the physical characteristics of the photo-detector array such as dimensions and the spacing between the array elements strictly depend on beam divergence.

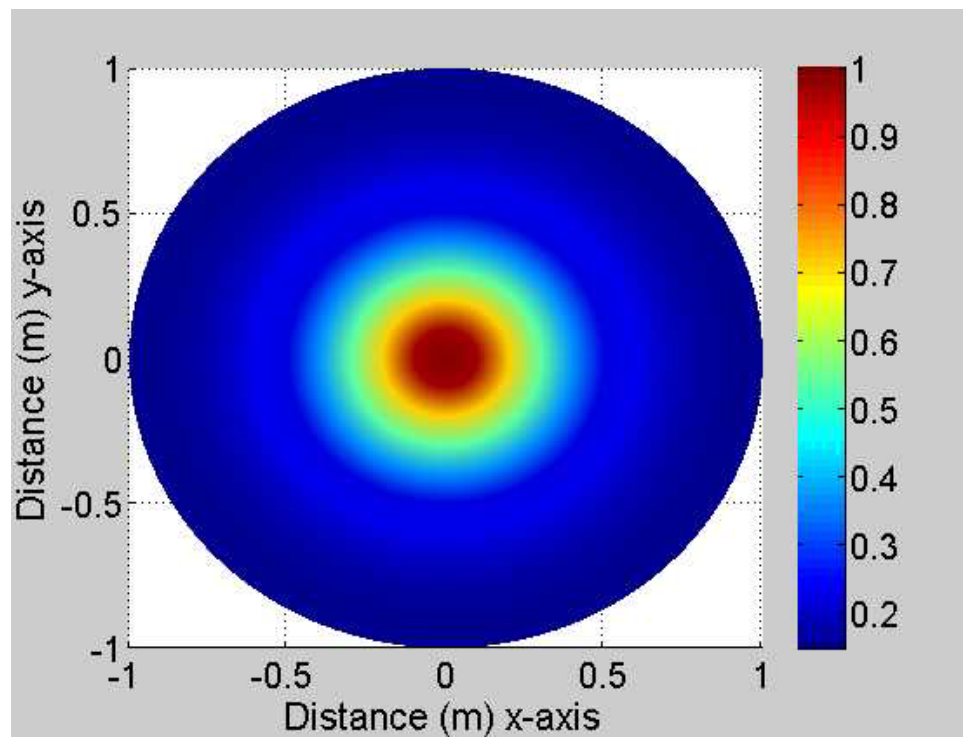

Figure 5: Plot of the cross-sectional beam pattern. The measurements were collected from 0 to $1.0 \mathrm{~m}$ at $\mathrm{x}$-axis and at $4.5 \mathrm{~m}$ at the illumination axis for 50W light source. The measurements between 500-550nm are averaged.

\section{DISCUSSION}

The study results provide valuable environmental information for modifying a photo-detector array design according to light field. According to the diffuse attenuation, a 500-550 nm band-pass filter will allow the observation at the light field from a single source as a 2-D Gaussian beam pattern. At this spectral range is around $0.1 \mathrm{~m}^{-1}$, the peak power of the beam (along the z-axis) will change from $100 \%$ to $23 \%$ as the array moves away from light from $4.5 \mathrm{~m}$ to a distance of $8.5 \mathrm{~m}$. The size of the beam pattern is a function of the divergence angle of the beam. In the current configuration, the FWHM radius expands from $0.3 \mathrm{~m}$ to $0.4 \mathrm{~m}$ as the array moves away from light from $4.5 \mathrm{~m}$ to a distance of $8.5 \mathrm{~m}$. The beam divergence can be modified using reflectors and optic elements in case more acute changes in the light field are needed over a shorter distance of $0.4 \mathrm{~m}$, the maximum length of the array.

During the empirical measurements in the UNH Tow Tank depth, several error sources were identified that limited an accurate correlation between the models and its corresponding measurements. These errorsincludedalignment errors andmeasurement errors underwater. Although the frame mounting all the elements was rigid and aligned, the internal alignment of the light source and of the detectorsmay not have been aligned perfectly along one axis. As a result, the profile measurementsof light along the z-axis and the along the xy-plane might be slightly skewed. Another factor is the water turbidity. An accurate calculation of the water turbidity in a survey site is very challenging.

Therefore, for more accurate distance detection algorithms, water turbidity should be taken into account.The focus of the current study emphasized 3-D translational motion. Future work will be towards expanding the research to characterize rotational motion. 
The study can be also applied in other applications, such as underwater optical communication and docking. Underwater optical communication can provide rates of up to $10 \mathrm{Mbits}$ over ranges of $100 \mathrm{~m}$ [14]. Several studies have investigated the use of omnidirectional sources and receivers in seafloor observatories as a wireless optical communication $[15,16]$. Another application is underwater docking by using optical sensors. Currently, studies have shown that such an application is possible for docking vessels as far as 10-15 meters for turbid waters and 2028 meters in clear waters [17].

\section{CONCLUSIONS}

Experimental work in this study was performed in order to evaluate the feasibility of a control design for underwater distance detection. The experiments included beam diagnostics, spectral analysis and intensity measurements using a 50Watt light source. A light source was mounted on a rigid frame to the wall in the tow tank and a light detector was placed underwater connected to a tow carriage that can move on rails along the tank. The study shows that a 500$550 \mathrm{~nm}$ band-pass filter will allow the observation of a light field from a single source as a 2-D Gaussian beam pattern. In the current configuration, the FWHM radius expands from $0.3 \mathrm{~m}$ to $0.4 \mathrm{~m}$ as the array moves away from light from $4.5 \mathrm{~m}$ to a distance of $8.5 \mathrm{~m}$. During the empirical measurements in the UNH Tow Tank depth, alignment errors and measurement errors underwater were identified that can limit the performance of the distance detection algorithms. In addition, the estimation of water clarity is a key parameter for the current distance detection algorithms. The focus of the current study took into account 3-D translational motion. Characterization of rotational motion between UUVs is planned in next steps of the research.

\section{ACK NOWLEDGEMENTS}

The authors would like to thank to BarbarosCelikkol, Yuri Rzhanov, Paul Levoie and Andy McLeod for their contributions to the technical discussions. Leslie Hubbard Marine Program Endowment is also greatly acknowledged for their financial support.

\section{REFERENCES}

[1] Acosta, G. G., Curti, H. J. and Calvo, O. A. (2005) "Autonomous underwater pipeline inspection in AUTOTRACKER PROJECT: the simulation module” Proceedings of IEEE-OCEANS 2005

[2] Inglis, G., Smart, C., Vaughn, I. and Roman, C. (2012) “A pipeline for structured light bathymetric mapping” 2012 IEEE/RSJ International Conference on Intelligent Robots and Systems October 7-12, 2012 Vilamoura, Algarve, Portugal

[3] D. B. Edwards, T. A. Bean, D. L. Odell and M. J. Anderson, (2004) "A leaderFollower Algorithm for Multiple UAV Formations,” Proceedings of 2004 IEEE/OES Autonomous Underwater Vehicles, Sebasco Estates, Maine, pp. 517-523, 2004.

[4] Jalbert, J, Baker, J., Duchesney, J., Pietryka, P., Dalton, W., Blidberg, D.R., Chappell, S., Nitzel, R., and Holappa, K., (2003) "A solar-powered autonomous underwater vehicle," Proceedings of OCEANS 2003, vol. 2, pp. 1132 - 1140, 2003.

[5] Hamilton, M., Kemna, S. and Hughes, D.T. (2010) “Antisubmarine warfare applications for autonomous underwater vehicles: The GLINT09 field trial results” Journal of Field Robotics, Vol. 27, No.6, pp. 890-902, 2010

[6] P. Colado and J. Sousa, "Leader-Follower Control of Underwater Vehicles over Acoustic Communications", Proceedings of 2011 IEEE OCEANS, Spain, June 2011. 
[7] S. Emrani and A. Dirafzoon and H. Talebi, "Leader Follower Formation Control of Autonomous Underwater Vehicles with Limited Communications", Proceedings of the IEEE International Conference on

ControlApplications, Denver, CO, pp.921-926, 2011.

[8] P. Feng, "Leader-Follower Cooperative Navigation with Communication Delays for Multi AUVs", Proceedings of the IEEE International Conferenceon Signal Processing, Communications and Computing (ICSPCC), Xi'an, China, 2011.

[9] Batista, B., Silvestre, C. and Oliviera, P. (2012) "GES Integrated LBL/USBL Navigation System for Underwater Vehicles” $51^{\text {st }}$ IEEE Conference on Decision and Control December 10-13, 2012 Maui, Hawaii, USA

[10] Eren, F., Thein, M.W, Celikkol B., DeCew J., Pe'eri, S. (2012) “Distance Detection of Unmanned Underwater Vehicles by Utilizing Optical Sensor Feedback in a Leader-Follower Formation" Proceedings of Marine Vehicle Autonomy MTS/IEEE 2012 Oct. 14-19, 2012 Hampton Roads, Virginia, USA

[11] Pe'eri, S. and Shwaery G. (2012) "Light field and water clarity simulation of neutral environments in laboratory conditions" SPIE Defense, Security and Sensing (Ocean remote sensing and monitoring IV), Baltimore MD, April 23 - April 26

[12] Moreno, I. and Sun, C.C. 2008 “Modeling the radiation pattern of LEDs” OSA Vol. 16, No.3 / OPTICS EXPRESS 1808

[13] C. Mobley, Light and Water, Academic Press Inc., 1994.

[14] Farr, N., Bowen, A., Ware, J., Pontbriand, C. and Tivey, M. (2010) “An integrated, underwater optical/acoustic communications system,” Proceeding of. OCEANS, May 2010, pp. 1-6

[15] Pontriband, C., Farr, N., Ware, J., Preisig, J. and Popenoe, H.(2008) “Diffuse High-Bandwidth Optical Communications” OCEANS 2008 , vol., no., pp.1-4, 15-18 Sept. 2008

[16] Farr, N., Chave, A., Freitag, L., Preisig, J., White, S., Yoerger, D. and Titterton, P.. (2005) “Optical modem technology for seafloor observatories” OCEANS, 2005.of MTS/IEEE, pages 928-934 Vol. 1, 2005

[17] Cowen, S., Briest, S. and Dombrowski, J. 1997, "Underwater Docking of Autonomous Undersea Vehicles Using Optical Terminal Guide” OCEANS ’97. MTS/IEEE Conference Proceedings San Diego, CA 